\title{
Risk Factors for Predicting Mortality among Old Patients with Acute Myocardial Infarction during Hospitalization
}

\author{
Liwei Chen, MD, Ling Han, MD, Jingguang Luo, MD \\ Department of Cardiology, Fuxing Hospital, Capital Medical University, Beijing 100038, China
}

\section{ABSTRACT}

Objective: To explore the risk factors to predict mortality among acute myocardial infarction (AMI) patients aged $\geq 80$ years during their hospitalization.

Methods: A cohort of AMI patients aged $\geq 80$ years hospitalized in our hospital were recruited from 2005 to 2014. Variables, including demographics, medical history, hemodynamic condition and laboratory results, were collected and analyzed to find risk factors predicting mortality during hospitalization.

Results: Amonga total of 384 patients enrolled in this study, the hospital mortality rate was $23.4 \%$. By multivariable stepwise regression analysis, nine independent significant predictors associated with mortality during hospitalization were identified: history of stoke, cardiac shock, Killip class III to IV, ventricular tachycardia, pulse rate $\geq 100 \mathrm{bpm}$ and $\mathrm{EF} \leq 40 \%$ at presentation, in-hospital bleeding, initial serum creatinine concentration $\geq 177 \mathrm{umol} / \mathrm{L}$ and elevated initial WBC level. Prior angina was identified as a protective factor. Hosmer-Lemeshow test indicated good fitness of the predicting variables.

Conclusion: Patients aged 80 and over have high mortality during hospitalization, and the risk prediction factors are clinically useful for predicting mortality in old patients with AMI.

\section{INTRODUCTION}

Prior studies have suggested that age is an important clinical risk predictor for mortality in patients with acute myocardial infarction (AMI). AMI continues to be an important cause of mortality, especially in elderly patients [Boucher 2001; Eagle 2004; Granger 2003; Hanna 2011]. The in-hospital mortality rates of old patients with AMI have exceeded 20\% [Mehta 2001; DeGeare 2000; Feldman 2006]. Thus, the old-patient cohort has become the most high-risk population, and has attracted more attention.

Received October 24, 2018; received in revised form December 21, 2018; accepted February 13, 2019.

Correspondence: Correspondence author: Ling Han, Department of Cardiology, Fuxing Hospital, Capital Medical University, Beijing 100038, China. Tel: 8610-88062089; E-mail: 552014173@qq.com
Numerous risk-prediction models for hospital mortality in patients with AMI have been developed from large randomized clinical trial populations, and effective risk stratification is integral to the appropriate therapy of patients with AMI [Krumholz 1999; Morrow 2000; Antman 2000; Boersma 2000; Tu 2001; Lee 1995; Addala 2004; De Luca 2004]. However, clinical risk predicting for AMI mortality in very old patients during hospitalization has seldom been reported. Therefore, this study aimed to explore the risk factors to predict mortality among AMI patients aged $\geq 80$ years during their hospitalization.

\section{METHODS}

\section{Study Design and Subjects}

This study was approved by Institutional Review Board and all patients provided informed consent. The overall study sample was restricted to patients aged $\geq 80$ years who were admitted from 2003 to 2012 at CCU department in Fuxing Hospital with a principal diagnosis of AMI (International Classification of Diseases, 10th Revision, ICD-10). ICD codes include I21.403, I21.902, I21.002, I21.001, I21.004, I21.103, I21.206-I21.208, I21.301 and I21.202. The exclusion criteria were patients transferred from another acute care facility, and patients whose AMI was diagnosed as an in-hospital complication. All the cases were confirmed AMI according to the ACC/AHA 2011 for NSTEMI and 2009 guidelines for STEMI [Anderson 2011; Kushner 2009].

\section{Data Collection}

From clinical experience and medical literature review, 34 candidate variables including demographic characteristics, medical history, clinical presentation and severity variables, and initial biochemical results were selected, and the data were collected from all patients. All variables followed standardized definitions. Left ventricular ejection fraction, hemoglobin level, admission SBP and HR were dichotomized and treated as binary variables. Severe left ventricular systolic dysfunction was defined as an LVEF $\leq 0.40$ [De Luca 2004]. According to WHO criteria and Chinese guideline, anemia was defined as a hemoglobin concentration below $110 \mathrm{~g} / \mathrm{L}$ in women and below $120 \mathrm{~g} / \mathrm{L}$ in men. SBP and HR were dichotomized by $90 \mathrm{mmHg}$ and 100 bpm, respectively [Eagle 2004; Granger 2003]. Creatinine 
Table 1. Patients' Baseline Characteristics

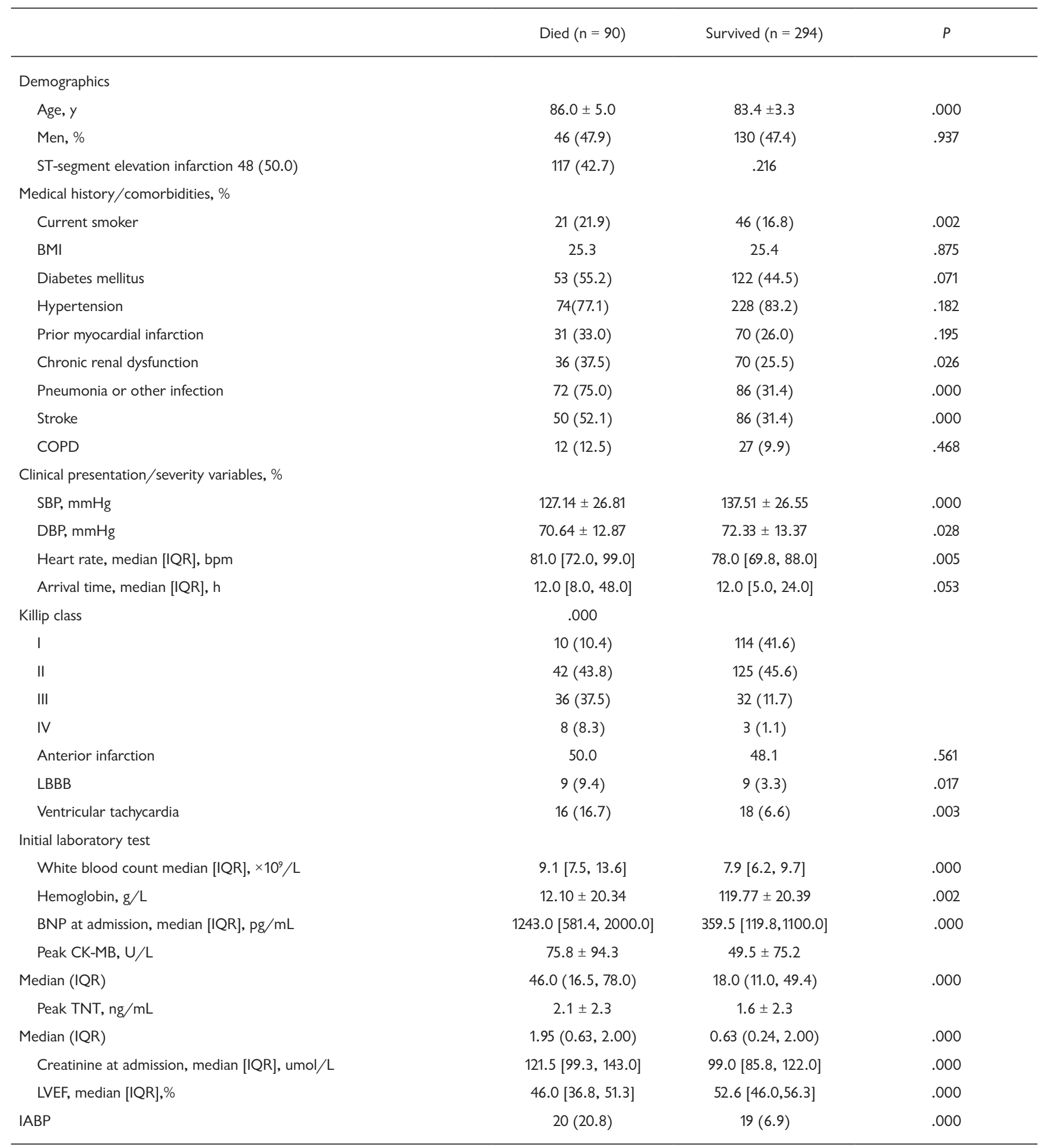

BMI indicates body mass index; COPD, chronic obstructive pulmonary disease; SBP, systolic blood pressure; DBP, diastolic blood pressure; LBBB, left bundlebranch block; AVB, second- or third-degree heart block; BNP, B-type natriuretic peptide; LVEF, left ventricular ejection fraction; IABP, intraaortic balloon counterpulsation. 
Table 2. Univariate Predictors of Hospital Mortality in AMI Patients

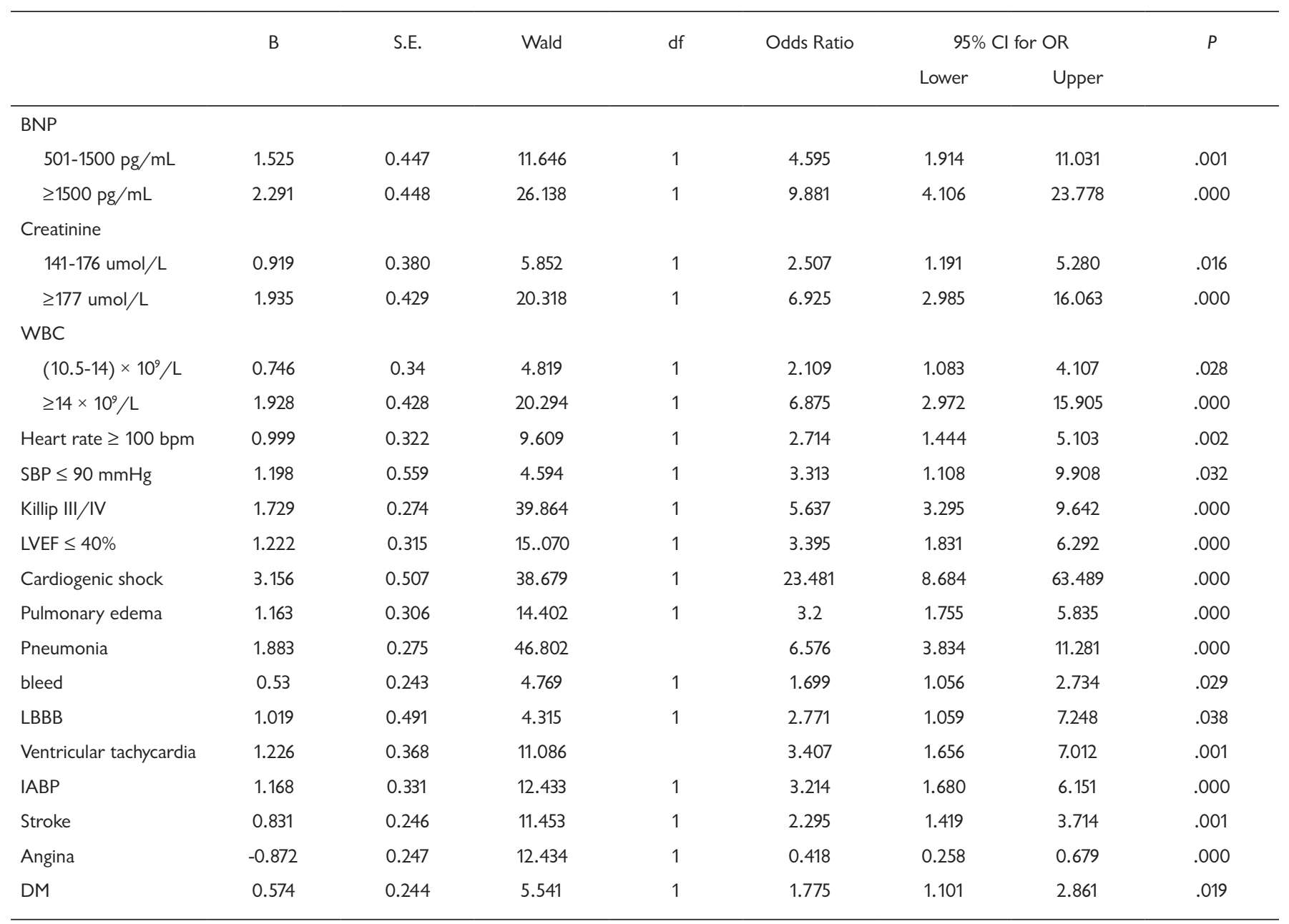

$\mathrm{Cl}$ indicates confidence interval; other abbreviations as in Table 1.

levels were grouped into four categories as $\leq 106 \mathrm{umol} / \mathrm{L}$, $107-140 \mathrm{umol} / \mathrm{L}, 141-176 \mathrm{umol} / \mathrm{L}$ and $\geq 177 \mathrm{umol} / \mathrm{L}$. BNP levels were grouped into four categories as $\leq 150 \mathrm{pg} / \mathrm{mL}$, $150-500 \mathrm{pg} / \mathrm{mL}, 501-1500 \mathrm{pg} / \mathrm{mL}$, and $\geq 1500 \mathrm{pg} / \mathrm{mL}$. WBC counts were grouped into four categories as $\leq 8.2 \times$ $10^{9} / \mathrm{L}, 8.2-10.5 \times 10^{9} / \mathrm{L}, 10.5-14 \times 10^{9} / \mathrm{L}$ and $\geq 14 \times 10^{9} / \mathrm{L}$ [Eagle 2004; Granger 2003].

\section{Statistical Analysis}

Baseline clinical characteristics and biochemical data were compared between the patients who died in hospital and the survivors. Continuous variables were presented as medians and interquartile ranges or mean $\pm \mathrm{SD}$ and compared using the nonparametric Mann-Whitney $\mathrm{U}$ test or two independent $t$ test. The Pearson chi-square test was used for dichotomous variables expressed as percentages. $P>.05$ was considered statistically significant. The univariate relationship between candidate variables and hospital mortality was determined by univariable logistic regression. Any predictor with a frequency of $<1 \%$ or $P>.05$ in univariable binary logistic regression was excluded. The relationship between the remaining baseline characteristics and mortality among hospital were analyzed by using multivariable logistic regression. Backward stepwise method was used (entry and exit criteria $P>.05$ and $P>.10$, respectively) to eliminate variables until only variables significant at the $P<.05$ level remained. The results were expressed as odds ratios with $95 \%$ confidence intervals.

\section{RESULTS}

\section{Baseline Characteristics of Patients}

This study enrolled 384 patients presenting with AMI; 90 patients died in hospital; and the hospital mortality rate was $23.4 \%$. Patients who died in hospital were older, more likely to have comorbidities such as diabetes mellitus, pneumonia or nosocomial infection, stroke and bleeding, more likely to have a lower SBP and LVEF, higher heart rate and Killip 
Table 3. Multivariable Predictors of Hospital Mortality in AMI Patients

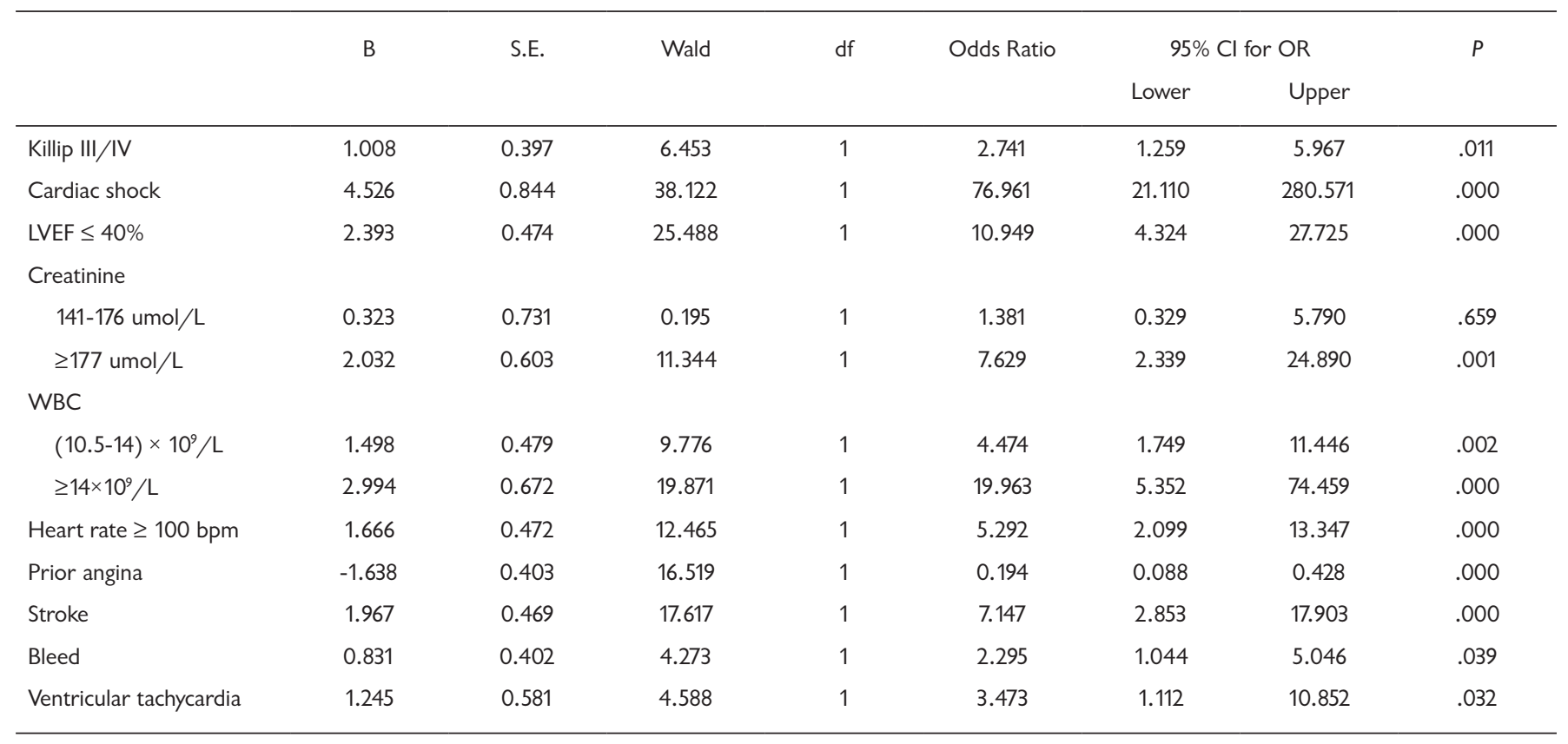

Abbreviations as in Table 1 and 2.

class, more pulmonary edema and cardiac shock at presentation, and had increased levels of WBC, creatinine, BNP and peak CK-MB. Furthermore, patients who died in hospital were less likely to have preexisting angina (Table 1).

\section{Univariate and Multivariate Analysis of Predictors of In- Hospital Mortality}

Significant univariate predictors of in-hospital mortality are listed in Table 2 . We identified 14 significant predictors for mortality including cardiogenic shock, Killip III/IV, pulmonary edema, pneumonia, IABP, BNP $\geq 1500$ $\mathrm{pg} / \mathrm{mL}$, creatinine $\geq 177 \mathrm{umol} / \mathrm{L}, \mathrm{WBC} \geq 14 \times 10^{9} / \mathrm{L}$ and $\mathrm{EF} \leq 40 \%$, while angina history appeared as a strong protective factor.

In contrast, the following candidate variables had no significant correlation with in-hospital mortality: creatinine $\leq$ $140 \mathrm{umol} / \mathrm{L}, \mathrm{WBC} \leq 10.5 \times 10^{\circ} / \mathrm{L}, \mathrm{BNP} \leq 500 \mathrm{pg} / \mathrm{mL}$, peak CK-MB and TNT, AVB, atrial fibrillation, Killip II, prior myocardial infarction, COPD, and the diagnosis of STEMI or NSTEMII.

Multivariable stepwise regression analysis showed that 10 independent significant predictors associated with inhospital mortality were history of stoke and angina, cardiac shock, Killip class III/IV, ventricular tachycardia, pulse rate $\geq 100 \mathrm{bpm}$ and $\mathrm{EF} \leq 40 \%$ at presentation, initial serum creatinine concentration $\geq 177 \mathrm{umol} / \mathrm{L}$, elevated initial WBC level, and in-hospital bleeding (Table 3). Using these variables, the Cox-Snell R square was 0.415 , and Nagelkerke R square was 0.624 , with a Hosmer-Lemeshow $P$ value of .076 (chi square: 12.871), which indicated good fitness of the predicting variables.

\section{DISCUSSION}

Although recent studies showed that age- and sex-adjusted 30-day mortality after myocardial infarction decreased from $10.5-18.9 \%$ to $7.8-16.1 \%$ during the past 10 years, the inhospital mortality in AMI patients aged 80 and over was still higher [Krumholz 2009]. It was reported that in-hospital AMI mortality rates were $23.2 \%$ in aged 80 to 84 and 26.3 $31.2 \%$ in aged 85 and over [Mehta 2001; DeGeare 2000; Feldman 2006]. AMI remains an important mortality factor for the oldest cohort. In our study, in-hospital mortality rate was $23.4 \%$, similar to the rates reported in previous studies.

In this study, 10 important independent risk predictors for in-hospital mortality in AMI patients aged 80 and over were identified. These variables were based on data from medical records obtained mainly at admission. The patients died in hospital had more co-morbidities, were more likely to develop renal insufficiency, pneumonia, stroke and bleeding, and presented more frequently with hemodynamic instability or cardiac shock. Our study further identified the prognostic importance of comorbidities and aggravated cardiac function in these patients. We found that cardiac shock was the single most powerful predictive variable of mortality, and baseline LVEF was a powerful determinant of mortality. Presenting with a low LVEF has been identified as a sign of incipient shock that might not be apparent at presentation [Webb 2000]. However, recently reported risk predictors have excluded LVEF from candidate variables [Morrow 2000; Boersma 2000].

Notably, our results showed the synergistic prognostic impact of both hemodynamic variables and comorbidities. 
Except for LVEF, we found some prognostic factors not commonly included in the studies of AMI patients, such as bleeding and elevated WBC. Elevated WBC is a marker of inflammatory process, which may increase the risk of mortality. The association between elevated WBC and adverse outcomes has been a focus of growing attention, but is rarely reported in risk predictor studies [Grzybowski 2004]. Interestingly, we found that prior angina was a protective factor against mortality during hospitalization. We speculated that protective function of prior angina may be due to ischemic preconditioning.

Unfortunately, the main limitation of our study is that the severity of coronary artery, an important predictor of mortality, was not collected. In our study, most of patients didn't receive coronary angiography due to their old age. Second, our analysis was limited to LVEF, WBC, and creatinine levels at admission. Therefore, we could not determine the effects of in-hospital LVEF, WBC, or creatinine levels on AMI mortality.

In conclusion, patients aged 80 and over have high inhospital mortality. We identified 10 important independent risk predictors (history of stoke and angina, cardiac shock, Killip class III to IV, pulse rate $\geq 100 \mathrm{bpm}$ and $\mathrm{EF} \leq 40 \%$ at presentation, ventricular tachycardia, initial serum creatinine concentration $\geq 177 \mathrm{umol} / \mathrm{L}$, elevated initial WBC level, and in-hospital bleed) for in-hospital mortality in AMI patients aged 80 and over. These risk factors are clinically useful for predicting mortality in patients with AMI. Knowledge of these risk factors should enhance clinical decision making with respect to further intervention in this cohort of patients.

\section{REFERENCES}

Addala S, Grines CL, Dixon SR, et al. 2004. Predicting mortality in patients with ST-elevation myocardial infarction treated with primary percutaneous coronary intervention (PAMI risk score). Am J Cardiol 93:629-32.

Anderson JL, Adams CD, Antman EM, et al. 2011. 2011 ACCF/AHA focused update incorporated into the ACC/AHA 2007 Guidelines for the Management of Patients with Unstable Angina/Non-ST-Elevation Myocardial Infarction: a report of the American College of Cardiology Foundation/American Heart Association Task Force on Practice Guidelines. Circulation 10:123:e426-579.

Antman EM, Cohen M, Bernink PJ, et al. 2000. The TIMI risk score for unstable angina/non-ST elevation MI: A method for prognostication and therapeutic decision making FREE. JAMA 284:835-42.

Boersma E, Pieper KS, Steyerberg EW, et al. 2000. Predictors of outcome in patients with acute coronary syndromes without persistent STsegment elevation. Results from an international trial of 9461 patients. The PURSUIT Investigators. Circulation 101:2557-67.

Boucher JM, Racine N, Thanh TH, et al. 2001. Age-related differences in in-hospital mortality and the use of thrombolytic therapy for acute myocardial infarction. CMAJ 164:1285-90.

DeGeare VS, Stone GW, Grines L, et al. 2000. Angiographic and clinical characteristics associated with increased in-hospital mortality in elderly patients with acute myocardial infarction undergoing percutaneous intervention (A pooled analysis of the primary angioplasty in myocardial infarction trials). Am J Cardiol 86:30-4.

De Luca G, Suryapranata H, van 't Hof AW, et al. 2004. Prognostic assessment of patients with acute myocardial infarction treated with primary angioplasty. Implications for early discharge. Circulation 109:2737-43.

Eagle KA, Lim MJ, Dabbous OH, et al. 2004. GRACE Investigators. A validated prediction model for all forms of acute coronary syndrome. Estimating the risk of 6-month postdischarge death in an international registry. JAMA 291:2727-33.

Feldman DN, Gade CL, Slotwiner AJ, et al. 2006. Comparison of outcomes of percutaneous coronary interventions in patients of three age groups ( $<60,60$ to 80, and $>80$ years) (from the New York State Angioplasty Registry). Am J Cardiol 98:1334-9.

Granger CB, Goldberg RJ, Dabbous O, et al. 2003. Predictors of hospital mortality in the global registry of acute coronary events. Arch Intern Med 163:2345-53.

Grzybowski M, Welch RD, Parsons L, et al. 2004. The association between white blood cell count and acute myocardial infarction in-hospital mortality: findings from the National Registry of Myocardial Infarction. Acad Emerg Med 11:1049-60.

Hanna EB, Chen AY, Roe MT, Wiviott SD, Fox CS, Saucedo JF. 2011. Characteristics and in-hospital outcomes of patients with non-ST-segment elevation myocardial infarction and chronic kidney disease undergoing percutaneous coronary intervention. JACC Cardiovasc Interv 4:1002-8.

Krumholz HM, Chen J, Wang Y, Radford MJ, Chen YT, Marciniak TA. 1999. Comparing AMI mortality among hospitals in patients 65 years of age and older: evaluating methods of risk adjustment. Circulation 99:2986-92.

Krumholz HM, Wang Y, Chen J, et al. 2009. Reduction in acute myocardial infarction mortality in the United States risk-standardized mortality rates from 1995-2006. JAMA 302:767-73.

Kushner FG, Hand M, Smith SC Jr, et al. 2009. 2009 focused updates: ACC/AHA guidelines for the management of patients with ST-elevation myocardial infarction (updating the 2004 guideline and 2007 focused update) and ACC/AHA/SCAI guidelines on percutaneous coronary intervention (updating the 2005 guideline and 2007 focused update) a report of the American College of Cardiology Foundation/American Heart Association Task Force on Practice Guidelines. J Am Coll Cardiol $54: 2205-41$.

Lee KL, Woodlief LH, Topol EJ, et al. 1995. Predictors of 30-day mortality in the era of reperfusion for acute myocardial infarction. Results from an international trial of 41,021 patients. GUSTO-I Investigators. Circulation 91:1659-68.

Mehta RH, Rathore SS, Radford MJ, Wang Y, Wang Y, Krumholz HM. 2001. Acute myocardial infarction in the elderly: differences by age. J Am Coll Cardiol 38:736-41.

Morrow DA, Antman EM, Charlesworth A, et al. 2000. TIMI risk score for ST-elevation myocardial infarction: A convenient, bedside, clinical score for risk assessment at presentation: an intravenous $\mathrm{nPA}$ for treatment of Infarcting Myocardium Early II Trial Substudy. Circulation 102:2031-7.

Tu JV, Austin PC, Walld R, Roos L, Agras J, McDonald KM. 2001. Development and validation of the Ontario acute myocardial infarction mortality prediction rules. J Am Coll Cardiol 37:992-7.

Webb JG, Sleeper LA, Buller CE, et al. 2000. Implications of the timing of onset of cardiogenic shock after acute myocardial infarction: a report from the SHOCK Trial Registry. Should we emergently revascularize occluded coronaries for cardiogenic shock? J Am Coll Cardiol 36:1084-90. 